\title{
A CLASS OF STRONG DIFFERENTIABILITY SPACES
}

\author{
JAMES B. COLLIER
}

ABSTRACT. It is shown that if the dual of a Banach space $X$ is weakly compactly generated, then each convex function on $X$ is Fréchet differentiable on a dense $G_{\delta}$ subset of its domain of continuity.

The domain of continuity of a convex function $f$ on a Banach space $X$ is the set of all points at which $f$ is continuous. If this set is nonempty, then $i t$ is equal to the interior of the convex set $\{x \in X \mid f(x)<+\infty\}$. The space $X$ is called a strong differentiability space (SDS) if each convex function on $X$ is Fréchet differentiable on a dense $G_{\delta}$ subset of its domain of continuity. Asplund first introduced this term in [1] and showed that $X$ is an SDS if $X^{*}$ is locally uniformly rotund or if $X$ is isomorphic to such a space. In particular, $X$ is an SDS if $X^{*}$ is separable. Troyanski [4] has observed that any reflexive space is an SDS. Phelps [3] has recently raised the question of whether $X$ is an SDS whenever $X^{*}$ is weakly compactly generated. We answer this question in the affirmative by using a result of Phelps [3]. First, we need a few preliminary definitions.

A Banach space $X$ is called weakly compactly generated (WCG) if there is a weakly compact subset whose linear span is dense in $X$. Thus $X$ is WCG whenever $X$ is separable or whenever $X$ is reflexive. If $f$ is a function on $X$ into $(-\infty,+\infty)$, the epigraph of $f$ is the subset of $X \times \mathbf{R}$ given by epi $f$ $=\{(x, r) \mid x \in X, r \geq f(x)\}$. We say that $f$ is convex if epi $f$ is convex, $f$ is proper if epi $f$ is nonempty, and $f$ is closed if epi $f$ is a closed subset of $X \times \mathbf{R}$. If $X \times \mathbf{R}$ is also a dual space, then we call $f$ weak*-closed if epi $f$ is a weak*-closed subset of $X \times \mathbf{R}$. For each proper convex function $f$ on $X$, there is a weak ${ }^{*}$-closed proper convex function $f^{*}$ on $X^{*}$, called the conjugate of $f$, given by

$$
f^{*}\left(x^{*}\right)=\sup \left\{\left\langle x, x^{*}\right\rangle-f(x) \mid x \in X\right\} .
$$

Let $C$ be a subset of $X, x \in C$, and $h$ a function on $C$ into $(-\infty,+\infty]$. If $h(x)=\sup \{h(z) \mid z \in C\}$, then we say that the supremum of $h$ over $C$ is attained strongly at $x$ if $h\left(x_{i}\right) \rightarrow h(x)$ implies that $x_{i} \rightarrow x$ for any sequnce $\left\{x_{i}\right\}$ in $C$. We call $x$ strongly exposed as a point of $C$ by a functional $x^{*} \in X^{*}$ if the supremum of $\left\langle\cdot, x^{*}\right\rangle$ over $C$ is finite and attained strongly at $x$. In this case, $x$ is also strongly exposed by $\lambda x^{*}$ for any $\lambda>0$. If, in addition, $X$ is

Received by the editors October 16, 1974 .

AMS (MOS) subject classifications (1970). Primary 46B99.

Key words and phrases. Fréchet differentiability, convex function, strongly exposed point. 
a dual space and $x^{*}$ is weak ${ }^{*}$-continuous, then $x$ is said to be weak*-strongly exposed. If $f$ is a function from $X$ into $(-\infty,+\infty]$, then we call $x$ a point of norm-rotundity of $f$ relative to $x^{*}$ if the supremum of $\left\langle., x^{*}\right\rangle-f$ over $X$ is finite and attained strongly at $x$. Norm-rotundity and strongly exposed points are related in the following way.

Lemma. Let $f$ be a function from a Banach space $X$ into $(-\infty,+\infty]$, $x \in X$, and $x^{*} \in X^{*}$. Then $f$ is norm-rotund at $x$ relative to $x^{*}$ if and only if $(x, f(x))$ is strongly exposed as a point of epi $f$ by $\left(x^{*},-1\right)$.

Proof. Suppose that $(x, f(x))$ is strongly exposed as a point of epi $f$ by $\left(x^{*},-1\right)$. Then $x_{i} \rightarrow x$ for any sequence of points $\left\{\left(x_{i}, a_{i}\right)\right\}$ in $X \times \mathbf{R}$ whenever $a_{i} \geq f\left(x_{i}\right)$ for all $i$ and $\left\langle\left(x_{i}, \alpha_{i}\right),\left(x^{*},-1\right)\right\rangle \rightarrow\left\langle(x, f(x)),\left(x^{*},-1\right)\right\rangle$. Hence, $x_{i} \rightarrow x$ if $\alpha_{i}=f\left(x_{i}\right)$ for all $i$ and $\left\langle x_{i}, x^{*}\right\rangle-\alpha_{i} \rightarrow\left\langle x, x^{*}\right\rangle-f(x)$. Thus $f$ is norm-rotund at $x$ relative to $x^{*}$. The converse follows similarly.

We now prove the main result.

Theorem. If $X$ is a Banach space and $X^{*}$ is WCG, then $X$ is an SDS.

Proof. Let $f$ be a convex function on $X$ with nonempty domain of continuity $D$. Choose a point $w \in D$ and an $\epsilon>0$, but sufficiently small so that $f(x)$ is bounded on the set $N=\{x \mid\|x-w\| \leq \epsilon\}$ and $N \subseteq D$. Define $g$ on $X$ by $g(x)=f(x)$ if $x \in N$, and $g(x)=+\infty$ otherwise. Then $g$ is a closed proper convex function on $X$, bounded on $N$, whose domain of continuity is the interior of $N$. We may assume without loss of generality that the unit ball $B$ is contained in $N$ and $-1 \leq g(x) \leq 0$ for all $x \in N$. Choose some $\lambda>1$ for which $N \subseteq \lambda B$.

Define $p$ on $X$ by $p(x)=0$ if $x \in B$, and $p(x)=+\infty$ otherwise. Define $q(x)=p(x / \lambda)-1$ for all $x \in X$. Clearly $p$ and $q$ are closed proper convex functions on $X$ and $q(x) \leq g(x) \leq p(x)$ for all $x \in X$. It follows from this by a well-known property of conjugate convex functions that $p^{*}\left(x^{*}\right) \leq g^{*}\left(x^{*}\right) \leq$ $q^{*}\left(x^{*}\right)$ for all $x^{*} \in X^{*}$. Some simple calculations show that

$$
\begin{gathered}
p^{*}\left(x^{*}\right)=\sup \left\{\left\langle x, x^{*}\right\rangle \mid x \in B\right\}=\left\|x^{*}\right\|, \\
q^{*}\left(x^{*}\right)=\sup \left\{\left\langle x, x^{*}\right\rangle+1 \mid x \in \lambda B\right\}=\lambda\left\|x^{*}\right\|+1
\end{gathered}
$$

for all $x^{*} \in X^{*}$. Thus $\left\|x^{*}\right\| \leq g^{*}\left(x^{*}\right) \leq \lambda\left\|x^{*}\right\|+1$ for all $x^{*} \in X^{*}$. These last inequalities show that the closed convex set $C=\left\{x^{*} \in X^{*}: g^{*}\left(x^{*}\right) \leq 2\right\}$ is bounded and has nonempty interior.

If we let $H=\left\{\left(x^{*}, r\right) \mid x^{*} \in X^{*}, r \leq 2\right\}$ and $C^{\prime}=$ epi $g^{*} \cap H$, then clearly $C^{\prime} \subseteq C \times[0,2]$. Hence $C^{\prime}$ is a bounded subset of $X^{*} \times \mathbf{R}$. Since $X^{*} \times \mathbf{R}$ is the dual of $X \times \mathbf{R}$, and epi $g^{*}$ and $H$ are both weak* -closed convex subsets, $C^{\prime}$ is a weak*-compact convex subset of $X^{*} \times \mathbf{R}$. Moreover, $X^{*} \times \mathbf{R}$ is WCG because $X^{*}$ is WCG. A result of Phelps [3] implies that $C^{\prime}$ is the weak*closed convex hull of its weak*-strongly exposed points. Consequently, there 
is a point $z^{*}$ in the interior of $C$ with $g^{*}\left(z^{*}\right)<2$ such that $\left(z^{*}, g^{*}\left(z^{*}\right)\right)$ is strongly exposed as a point of $C^{\prime}$ by some functional $(z,-1) \in X \times \mathbf{R}$.

Since $g^{*}\left(z^{*}\right)<2$ and epi $g^{*}$ is convex, $\left(z^{*}, g^{*}\left(z^{*}\right)\right)$ is also strongly exposed as a point of epi $g^{*}$ by $(z,-1)$. The Lemma implies that $g^{*}$ is normrotund at $z^{*}$ relative to $z$. From this it follows by Theorem 1 in [2, p. 450] that $g$ is Fréchet differentiable at $z$ with Fréchet gradient $z^{*}$. Clearly for this to be true, $z$ must lie in the interior of $N$. Thus $f$ is also Fréchet differentiable at $z$ and $\|z-w\|<\epsilon$.

Since the choice of $w \in D$ and $\epsilon>0$ was arbitrary, the set $G$ of points at which $f$ is Fréchet differentiable must be dense in $D$. Since $G$ is dense, it follows from Lemma 6 in $[1$, p. 43$]$ that $G$ is, in fact, a dense $G_{\delta}$ subset of $D$. Therefore, $X$ is an SDS.

Remark added in proof. Since the submission of this article for publication, the author has learned that I. Namioka and R. R. Phelps have found an independent proof of the main result.

\section{REFERENCES}

1. E. Asplund, Fréchet differentiability of convex functions, Acta Math. 121 (1968), 31-47. MR 37 \#6754.

2. E. Asplund and R. T. Rockafellar, Gradients of convex functions, Trans. Amer. Math. Soc. 139 (1969), 443-467. MR 39 \#1968.

3. R. R. Phelps, Dentability and extreme points in Banach spaces, J. Functional Anal. 17 (1974), 78-90.

4. S. L. Trojanski, On locally uniformly convex and differentiable norm. in certain non-separable Banach spaces, Studia Math. 37 (1970/71), 173-180. MR 46 \#5995.

DEPARTMENT OF MATHEMATICS, UNIVERSITY OF SOUTHERN CALIFORNIA, LOS ANGE LES, CALIFORNIA 90007 